\title{
The Role of Jupiter in Chondrule Formation
}

\author{
J.-D. BODENAN ${ }^{1,2}$, C. SURVILlE ${ }^{2}$, J. SZULAGYI $^{2}$, L. \\ MAYER $^{2}$, M. SCHÖNBÄCHLER ${ }^{1}$ \\ ${ }^{1}$ Institut für Geochemie und Petrologie, Departement \\ Erdwissenschaften, Clausiusstrasse 25, 8092, Zürich, \\ Switzerland. (jean-david.bodenan@erdw.ethz.ch;) \\ ${ }^{2}$ Institute for Computation Science, Winterthurerstrasse 190, \\ 8057, Zürich, Switzerland
}

Chondrule textures and chemistry require formation in flash-heating events and subsequent relatively rapid cooling. They, however, retained $\mathrm{Na}$, which should have been lost during such events, implying that they formed in an environment with high gas pressure or high dust/gas ratio [1].

We used the 2D hydrodynamical code RoSSBi to simulate the effects of a Jupiter-like planet such as shocks on the disk to evaluate whether they could generate environments for chondrule formation.

The simulations show that the interaction between the planet and the disk creates a region of low densities around the planet's orbit. It is coherent with gaps observed in protoplanetary disks [2] and limits the exchange of material between inside and outside the gap. This can serve as an explanation for the isotopic dichotomy observed between carbonaceous chondrite-related material in the outer solar system and that linked to ordinary and enstatite chondrite found further in [e.g. 3].

The difference of velocities between the planet and the gas generates shocks that propagate both in- and outwards through the disk. Another consequence of the planet's interaction is the formation of large vortices ( $\sim 1 \mathrm{AU}$ in size $)$ that concentrate particles and gas, leading to high gas pressure and enhanced gas and dust density.

The shocks create elevated temperatures $(\mathrm{T})$ in the disk. However, whether $\mathrm{T}$ sufficient to melt these precursors can be achieved, strongly depends on the planet mass and disk properties such as its mass, the cooling mode, or its metallicity.

\section{References}

[1] Alexander C. M. O'D. et al. (2008) Science 320:16171619.

[2] Fedele D. et al. (2018) Astronomy and Astrophysics 610:A24.

[3] Leya et al. (2008) Earth and Planetary Science Letters 238:233-244. 